\title{
Kann man ohne Brett auf der nächsten Welle surfen?
}

\author{
Ein Interview mit Meinrad Perrez
}

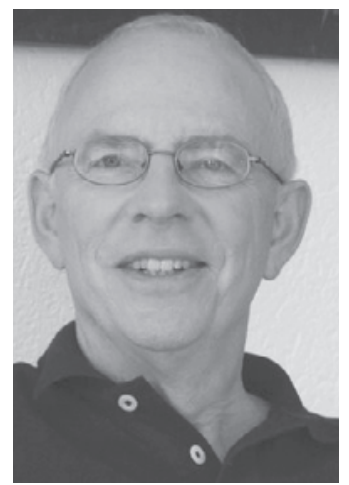

Professor Meinrad Perrez ist als Klinischer Psychologe einer der ersten und wichtigsten Vertreter der Verhaltenstherapie im schweizerischen Universitätsumfeld. Er war von 1973 bis 1975 Professor für Psychologie an der Freien Universität Berlin, nachdem er zuvor bereits an der Universität Salzburg erste Vorlesungen zur Verhaltenstherapie gehalten hatte. Von Berlin wechselte er 1975 an die Universität Fribourg (Schweiz), wo er unter anderem die Akademie für Verhaltenstherapie bei Kindern und Jugendlichen an den Universitäten Basel, Fribourg und Zürich und das interfakultäre Institut für Familienforschung und -beratung an der Universität Fribourg mitbegründete. Professor Meinrad Perrez teilt die Sorge nicht weniger Kolleginnen und Kollegen, dass die Verhaltenstherapie zu einer Therapieform werden könnte, die als solche gar keine verhaltenstherapeutische Identität mehr besitzt, sondern von immer neuen therapeutischen Wellen zur Unkenntlichkeit verwaschen wird. Aus diesem Grund wurde von meinem Herausgeberkollegen Professor Fritz Hohagen folgende Frage gestellt: "Kann man ohne Brett auf der nächsten Welle surfen?» Das Gespräch führte Prof. Dr. Ulrike Ehlert, Zürich.

Lieber Herr Professor Perrez, Sie sind einer der ersten Psychologen in der Schweiz gewesen, der sich engagiert wissenschaftlich und praktisch für die Etablierung der Verhaltenstherapie eingesetzt hat. Wie sehen Sie rückblickend die Entwicklung der Verhaltenstherapie im deutschsprachigen Raum?

Ihre Geschichte im deutschsprachigen Raum ist einerseits eine Erfolgsgeschichte und andererseits werden ihre Konturen undeutlicher. Das halte ich teilweise für eine Folge ihres Verständnisses als ein offenes, am wissenschaftlichen Lernen orientiertes System, das sich in Richtung der Psychotherapie als «Psychologische Therapie» entwickelt, weg von den Schulen. Der Verlust ihrer Konturen ist aber auch durch die Neigung mitbedingt, nachfrageorientiert auf der nächsten Welle zu surfen, ohne solides Brett unter den Füßen, wie Sie es metaphorisch trefflich angesprochen haben!

\section{Wo liegen die Erfolge der Verhaltenstherapie?}

Ich sehe sie zunächst im gewachsenen Verständnis psychischer Störungen und in der erfolgreichen Entwicklung eines
Methodenrepertoires, das intensiver Forschung in seiner Funktionsweise und Wirksamkeit unterzogen wird. Dann sehe ich sie im Faktum, dass dieser psychotherapeutische Ansatz heute in den meisten industrialisierten Ländern der Welt präsent ist. In den meisten Ländern der westlichen Welt gibt es darüber hinaus unterdessen etablierte Weiterbildungsinstitutionen. Dass im deutschsprachigen Europa die Verhaltenstherapie (VT) weitgehend an den Universitäten unterrichtet wird und die Weiterbildungsstudiengänge dort angesiedelt sind, halte ich für einen besonderen Fortschritt.

\section{Worin besteht dieser Fortschritt?}

Die enge Andockung der VT an die Forschung ist für mich, wie vorher angedeutet, ein Wesenselement der VT. Diese Vernetzung ist ein protektiver Faktor dafür, dass die VT nicht in Sekten abgleitet, sondern im weiten Sinn als jener psychotherapeutische Ansatz verstanden wird, der zur Lösung von psychischen Problemen das verfügbare theoretische, empirische und methodische Wissen der psychologischen Forschung und ihrer Nachbardisziplinen einsetzt, so wie sie Hans Rein-

\section{KARGER}

Fax +497614520714

Information@Karger.de

www.karger.com

\section{(c) 2011 S. Karger GmbH, Freiburg}

Accessible online at:

www.karger.com/ver
Prof. Dr. Meinrad Perrez

Departement für Psychologie

Universität Fribourg

Rue de Faucigny 2, 1700 Fribourg, Schweiz

Tel. +41264025634

meinradperrez@bluewin.ch 
ecker bereits in den 1980er Jahren unter Bezugnahme auf M.B. Shapiro charakterisiert hat.

Ein solches Programm ist ein offenes System, das gegen therapeutische Kirchen und Kapellengründungen besser geschützt ist. Man kann sich sogar fragen, worin sich ein so definierter Ansatz vom Programm der Klinischen Psychologie unterscheidet, soweit es die Ätiologie und Therapie betrifft. Dieses offene System hat auch dazu geführt, dass in den letzten Jahrzehnten nicht nur kognitive verhaltensrelevante Aspekte in die VT integriert worden sind, sondern auch endokrinologische, wozu unter anderem Zürich oder Trier viel beigetragen haben, und neurobiologische störungs- und therapierelevante Faktoren das Verständnis und die Therapie mehrerer Störungen verbessert haben.

Braucht es überhaupt noch die aus der experimentellen Lernpsychologie abgeleiteten therapeutischen Verfahren?

Die Lernpsychologie wird für jede Psychotherapie notwendigerweise eine zentrale Rolle spielen. Wenn durch psychotherapeutische Erfahrung Verhaltensdispositionen, Dispositionen des Wahrnehmens, Bewertens, des Fühlens und Denkens mehr oder weniger stabil verändert werden - das bleibt wohl zentrales Ziel von Psychotherapie -, so findet definitionsgemäß Lernen statt. Deshalb ist und bleibt die Erforschung des Lernens ein Kernelement der Psychotherapieforschung. Psychotherapie - welcher Provenienz auch immer - besteht in der Inszenierung von Lernprozessen; wenn sie erfolgreich ist, so hat Lernen stattgefunden, gleichgültig welche wissenschaftlichen oder esoterischen Kausalattributionen hierfür bemüht werden. Eine Psychotherapie wie die VT, die wissenschaftlich orientiert ist, wird die wissenschaftliche Analyse der involvierten Lernprozesse im Zentrum des therapeutischen Forschungsinteresses behalten.

Waren wir in den 1970er Jahren des letzten Jahrhunderts stolz darauf, verhaltenstherapeutische Standardverfahren zu erlernen und anzuwenden, scheint heute das Bedürfnis der Weiterbildungsteilnehmerinnen und -teilnehmer darin zu bestehen, möglichst neue Therapiebausteine wie beispielsweise «mindfulness therapy» oder Schematherapie zu erlernen. Was halten Sie von dieser Entwicklung?

Es könnten unter anderem noch die «acceptance and commitment therapy», die «dialektische Verhaltenstherapie» und andere ergänzt werden. Man hat den Eindruck, dass jeder, der es in dieser Domäne zu etwas bringen will, eine neue Therapie erfindet. Ich habe die «Intuitionstherapie» erfunden, um auf die Fragwürdigkeit der inflationären Methodenkreation aufmerksam zu machen (dargestellt in unserem «Lehrbuch Klinische Psychologie-Psychotherapie»)! Die Halbwertszeiten für die einzelnen Wellen scheinen kürzer zu werden.

Als Klinischer Psychologe und Verhaltenstherapeut gehe ich davon aus, dass das Inszenieren von psychotherapeuti- schen Lernprozessen zwar einen breiten Handlungsspielraum offen lässt. Von den durch die Verhaltenstherapie ritualisierten Inszenierungen erwarten wir indes, dass sie wissenschaftlich fundiert sind. Dazu gehört neben der theoretischen Einbettung von Ätiologie und Therapie in den rationalen Korpus der akademischen Psychologie auch der empirische Nachweis ihrer Wirksamkeit. Die theoretische und empirische Fundierung des therapeutischen Handelns sind das Brett, auf dem Verhaltenstherapeuten surfen!

Das muntere Sprießen und Vermarkten neuer VT-Methoden erstaunt mich, wenn sie in Weiter- und Fortbildungsveranstaltungen vermittelt werden, bevor ihre Fundierung seriös geklärt ist. Zu einzelnen der neuen VT-Methoden gibt es indes nennenswerte Forschung.

\section{Was erwarten Sie sich für die Zukunft der Psychotherapie?}

Die aktuelle klinisch-psychologische Forschung setzt sich zunehmend mit der Frage auseinander, wie psychische Störungen funktionieren. Und wenn ich die Forderung teile, dass therapeutische Methoden als wissenschaftlich fundierte Verfahren zur Linderung von psychischen Störungen ihren Wirkungsnachweis erbringen müssen, so erwarte ich dennoch die großen Fortschritte der Therapie im besseren Verständnis des psycho-biologischen Funktionierens von Menschen mit psychischen Störungen. Hätte sich die Medizin im 19. und 20. Jahrhundert in ihrer Forschung auf den Wirkungsnachweis der damals verfügbaren Methoden konzentriert, so wüssten wir heute, welche Effektstärken - ich sage es etwas karikierend - gewisse Arten von Tee, der Aderlass oder KneippMethoden für die Heilung gewisser Störungen im Vergleich zu anderen Methoden haben.

\section{Die Erkenntnisse über das Funktionieren haben die Therapie weitergebracht.}

Auch wenn in den letzten Jahren die Begeisterung für die bildhaft so schön darstellbare Lokalisierung der Gehirnaktivität anlässlich psychischer Leistungen in ihrem Erkenntnisgewinn von einer mächtigen Überschätzung getragen war, so stellen doch die Forschungen der Neurobiologie, der Genetik und der Endokrinologie eine durch die Psychologie nicht mehr ignorierbare Bereicherung und Vertiefung des Verständnisses menschlichen Erlebens und Verhaltens dar. Die Klinische Psychologie der Zukunft wird sich nicht in Neurobiologie, Endokrinologie oder Genetik auflösen, aber sie wird biologisch informiert sein und gewisse psychologische Fragen werden bessere Antworten finden, wenn wir das Zusammenspiel der verschiedenen Systeme besser verstehen.

Neben der Verbesserung des Verständnisses der Ätiologie und des Funktionierens psychischer Störungen erwarte ich auch eine fruchtbarere Nutzung der methodischen Entwicklungen der Datengewinnung für die Forschung und die Diagnostik in der Praxis, wurden doch in den letzten Jahren nicht 
nur neue multimodale Zugänge zur Beobachtung und Registrierung von Verhalten und Erleben im Labor, sondern besonders auch in der natürlichen Umgebung entwickelt und erprobt, die in der Praxis bisher weitgehend ignoriert werden, obwohl sie gerade für die Verhaltenstherapie theoretisch kongenial sind. Ich denke hier an das «Ambulante Assessment». Kurt Pawlik und Lothar Buse haben in den 1980er Jahren einmal zur Psychologie als Wissenschaft des Verhaltens - mit Bezug auf Karl Bühler, der die Psychologie der 1930er Jahre als eine Wissenschaft der Seele ohne Seele bezeichnet hatte - moniert, dass ihr das Verhalten abhanden gekommen ist. Diese Gefahr ist auch in der verhaltenstherapeutischen Praxis zu beobachten, insofern sie ihre Datenquellen und -methoden auf Fragebogendaten und den retrospektiven Selbstbericht einschränkt.

Wie sehen Sie in diesem Zusammenhang die aktuellen Klassifizierungsmodalitäten?

Eine angemessene störungsbezogene Diagnostik erfordert eine angemessene Konzeption psychischer Störungen. Von den Bollwerken der Klassifikation psychischer Störungen, vom ICD und DSM, erwarte ich, dass diese auf Expertennormen basierenden phänomenologischen Klassifikationssysteme - die alle paar Jahre per Expertenbeschluss durch Verfeinerung der Kriterien und durch Hinzufügung neuer und Streichung alter Kategorien revidiert werden -, von ihrer künftigen Entwicklung erwarte ich, dass die Zukunft forschungsbasierten Ordnungssystemen gehört, deren Validität auf den Ergebnissen der psychologischen und biologischen Forschung beruhen wird. Das Problem wird unter anderem drastisch manifest bei der Klassifikation der Persönlichkeitsstörungen, die am eindrücklichen Korpus der jahrzehntelangen erfolgreichen psychologischen Persönlichkeitsforschung vorbei munter Kategorien erfindet und streicht. Für die nächste DSM-Runde soll eine Halbierung der Anzahl diskutiert werden!

Die diesen Ordnungssystemen inhärente Lösung, die Komplexität der in Erscheinung tretenden Probleme durch Komorbiditätsdiagnosen abzubilden, hat eine fragwürdige Fraktionierung der Person zur Folge, die der Entdeckung der möglichen inneren Organisation der Symptome im Weg steht.
Eine manualisierte Therapieforschung, deren Manuale sich primär an den gerade gängigen Klassifikationssystemen orientieren, findet ihre Grenzen an der Fragwürdigkeit der Klassifikation. Diese Problematik stellt sich für verschiedene Störungsbereiche in unterschiedlichem Ausmaß. Widiger und Trull [2007] haben in ihrem Beitrag im American Psychologist wichtige Mängel zusammengefasst: exzessiv häufige Komorbiditätsdiagnosen, unzureichende Abbildung der Verhaltensvariabilität, arbiträre Abgrenzung von normalem Verhalten und Heterogenität der Personengruppen mit der gleichen Diagnose. Ich würde noch hinzufügen: Nicht-Inanspruchnahme der methodischen Möglichkeiten der modernen Diagnostik auf den Ebenen der Datenerfassung und der Psychometrie.

\section{Was wünschen Sie der VT für die Zukunft?}

Sowohl der «Sache der psychischen Störungen» als auch der Klinischen Psychologie wünsche ich mehr Aufmerksamkeit für die störungsspezifische ätiologische Forschung auf der Basis valider, forschungsbasierter Störungseinheiten. Je besser wir das individuelle Funktionieren verstehen, umso erfolgreicher werden wir therapieren.

Der verhaltenstherapeutischen Praxis wünsche ich eine Verstärkung der therapeutischen Identität als Anwender wissenschaftlich fundierter Erkenntnisse und eine mutigere Verwendung neuer technischer Möglichkeiten in der Informationsgewinnung und Diagnostik und in der Therapie. Ich denke unter anderem an die Nutzung von «Ambulantem Assessment» für die Verhaltensdiagnostik sowie an die Unterstützung des therapeutischen Handelns durch Nutzung virtueller Realität, Internet-basierter und Computer- sowie Mobiltelefon-unterstützter Interventionen.

Herr Professor Dr. Meinrad Perrez, ich danke Ihnen für dieses Gespräch.

\section{Literatur}

Widiger TA, Trull TJ: Plate tectonics in the classification of personality disorder: shifting to a dimensional model. Am Psychol 2007;62:71-83. 